\title{
A Low Cost Posture Monitoring System Based on an Ambient Light Sensor Network
}

\author{
Yu-Jia Sun, Ji-Yan Yu, Xiao-Ming Wang \\ School of Mechanical Engineering \\ Nanjing University of Science and Technology \\ Nanjing, China \\ E-mail: sunyujia1989@gmail.com
}

\author{
Neil Nergmann \\ School of Information Technology and Electrical \\ Engineering, University of Queensland \\ Brisbane, Australia \\ E-mail: n.bergmann@itee.uq.edu.au
}

\begin{abstract}
This paper applies wireless sensor networks to monitoring sitting condition (presence and posture). Specifically, we develop a sitting condition monitoring system based on an ambient light sensor network. As the system is hard to be modeled, a feature learning experiment has been conducted to learn about the features to design a classifier. We conducted an evaluation experiment in five different environments. Our experiment results show that our system has an accuracy around $82 \%$, and it is robust to five different environmental noise.
\end{abstract}

Keywords-sensor networks; sitting condition; monitoring

\section{INTRODUCTION}

With an increasing proportion of the population working in sedentary occupations, there have been an increase in the proportion of back and spinal injuries associated with poor posture [1]. A variety of ergonomic systems have been proposed to improve the sitting conditions such as a smart chair based system [2]. Active interventions to remind users of poor posture have proven beneficial [3]. In [4], a smart chair system with in-built sensors can provide such feedback to workers with an accuracy of about $80 \%$, but the hardware and computation cost is relatively high. [5] presents a visual system for posture classification based on Kinect ${ }^{\mathrm{TM}}$, which demonstrates that such a system can be used efficiently monitor a person's posture. However video-based systems also involve relatively high cost and have potential privacy implecations. Other methods based on accelerometers, smart glasses and smart phonse have also been proposed [6,7]. The accuracy of the wearable device based methods is not as good as camera based methods, but the cost is lower and setup is convenient. To encourage wider adoption, a very simple and inexpensive system which retains good accuracy is preferred.

In this paper, we present a sitting condition monitoring system based on a wireless ambient light sensor network, which detects presence/absence and good/poor sitting posture. Using just two light sensors, and a machine-learning approach to optimize the system classification accuracy, a classification accuracy of $82 \%$ is achieved.

In section 2, the sensing system is described. In section 3, an experimental setup and its associated classifier learning as well as results of testing are described, and section 4 outlines our consclusions and future work.

\section{METHODS}

An ambient light sensor measures the intensity of visible light. In our system, we set up an ambient light sensor network which "looks" at the subjects sitting in front of a desk. A set of training examples is then used to decide upon suitable sensor features, and to train a classifier based on those features.

Light sensor classification will depend on the location of the lighting sources and good placement of the sensors. In this experiment, the lighting source is natural light from a window, as shown in Figure 1. Depending on the presence and position of the subject, the light received by the sensors will vary, as shown in Fig. 2 . The received power of the sensors is P1 and P2 respectively, and the angle of bend of the subject's posture is $\theta$, as shown in Fig. 3. As $\theta$ varies, so do the relative values of $\mathrm{P} 1$ and $\mathrm{P} 2$, as shown in Fig. 2. The subject is asked to sit in a good posture for a few seconds and then sit in a poor posture. The valley in Fig. 2 is caused by a poor sitting posture. Based on the variation of $\mathrm{P} 1$ and $\mathrm{P} 2$ with posture, the proposed system should be able to be used to monitor a subject's sitting condition.

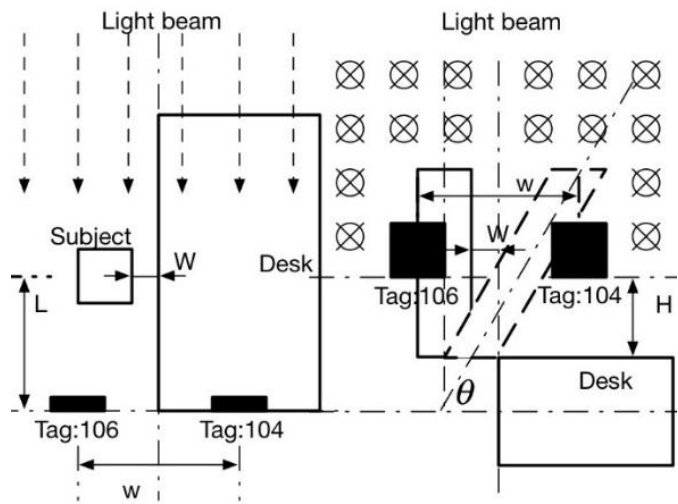

$\begin{array}{ll}\text { (a) Vertical view } & \text { (b) Front view }\end{array}$

Figure 1. Scenarios of an ambient light sensor network method.

To train the classifier and to evaluate the performance of our system, we need labelled training and test data. This is done by filming all the experimental sessions and manually labelling data.

We also wish to compare this approach against other approaches, and so a camera based contour matching algorithm is used to also classify posture and presence. We 
attach three marker labels on the subject's wrist, shoulder and face. We use contour matching method to get the position of the marker labels on each video frame. Given each label's position, we can accurately calculate the angle $\theta$. Then these angles will be used to categorize the subject's posture. This video-based classification will be compared to light-based classification in the evaluation section later.

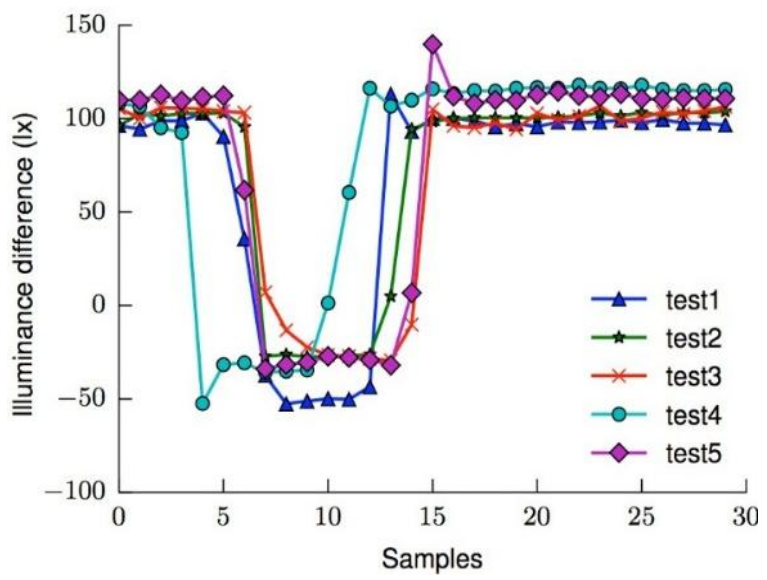

Figure 2. Illuminace difference with good posture and poor posture

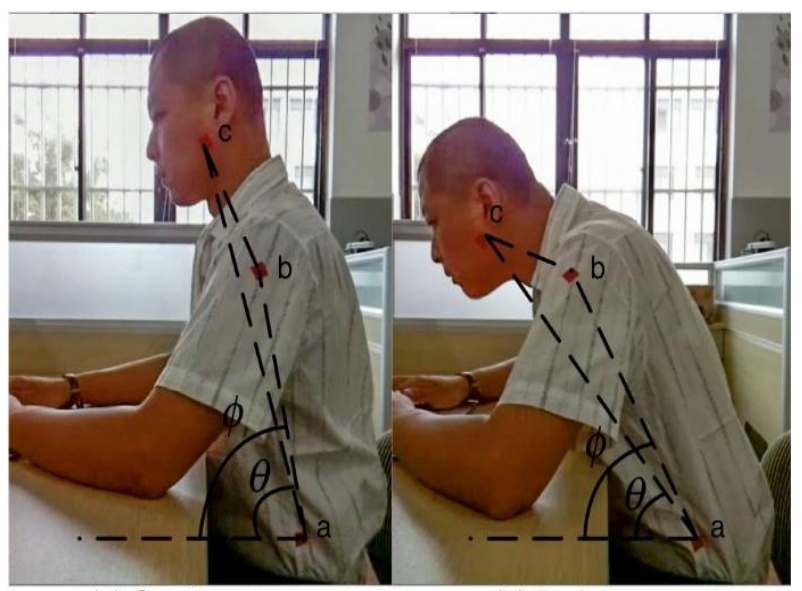

(a) Good posture

(b) Bad posture

Figure 3. Sitting posture

\section{EXPERIMENT}

Absolute values from the light sensors depend not only on the subject's posture but also on the strength of the incoming light from the window. If we place the sensors so that one sensor is shadowed by a subject with poor posture, and the other sensor is placed to be relatively unaffected by posture, then the difference in light power between the sensors gives a significant, repeatable response to poor posture, as shown in Fig. 2.

The first stage of our classifier design is to decide on a good set of features which can give reliable classification, and then to train a classifier based on those features,

In the second stage, the performance of our system is evaluated against the camera based method in five different environments to analyse the accuracy and the robustness of our system.

\section{A. System platform and setup}

The intensity of visible light around the subject was collected using OPT3001 ambient light sensors from Texas Instruments. The OPT3001 is a single-chip lux meter, and measurements can be made from 0.01 lux up to $83 \mathrm{k}$ lux without manual range selection and the maximum resolution is 0.01lux. The light sensors were mounted to SensorTag2.0 boards, which sampled the sensors at up to $10 \mathrm{~Hz}$ (this sample rate can be set manually) and the data is transmitted to a central computer wirelessly through Bluetooth. Various sampling and transmission rates were tried, and a rate of $1 \mathrm{~Hz}$ was used during the experiments. During the experiment, the SensorTag2.0 is powered by an USB cable which gives continuous operation. The SensorTag2.0 can also be powered by a CR2032 battery for roughly 10 hours using our software. The central computer is a Raspberry Pi 3 model B (RPi3) board running the Rapbian operating system with an onboard Bluetooth chip. A Python script used to collect light data and classification was done with another Python script. To compare our method based on light sensor with the camera based method, we also attached a CSI camera board to the RPi3 board. A Python script running a contour matching algorithm will be used to label the subjects' sitting posture through this CSI camera board. The setup of the system is shown in Figure 4. As the data is fetched and processed on our central computer in real time, there is no need to add time labels to the sensor data, and sensor node time synchronization is not needed.

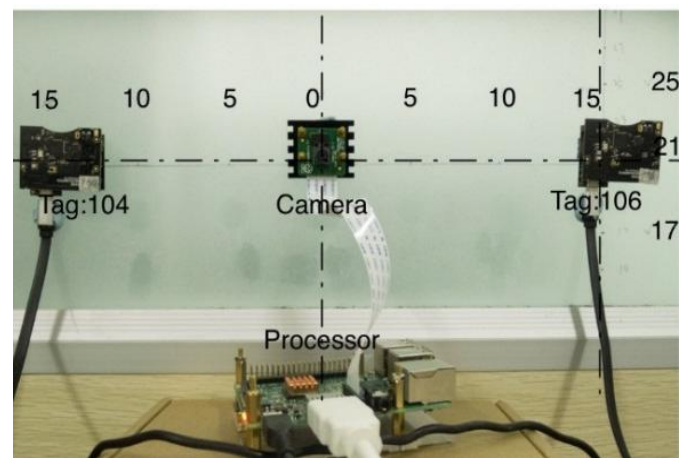

Figure 4. Setup of the proposed system

\section{B. Feature learning}

In this system, four features are chosen to do the recognition. The absolute amplitude of the light signal from two different nodes is indicated by $\delta_{1}$ and $\delta_{2}$. The amplitude of the difference signal of the light is indicated by $\rho$ and the duration of a low value of difference in indicated by $\tau$. A typical signal is shown in Figure 5. During training, the signal is separated into five phases of five different conditions. The meaning of the labels in Figure 5 is shown in Table I. During the phases of F2 and F3, the amplitude of the light signal difference $(\rho)$ changes from negative (about -50 lux) to positive (about +100 lux), which can be used to detect a poor posture phase. In some cases, the subject legitimately be leaning forward to pick up a book or a pen, and this should not be labelled as poor posture. This short period action means poor posture cannot be determined just by light 
difference. So the time duration $(\tau)$ of negative difference is to help to exclude this noisy action. The features $\delta_{1}$ and $\delta_{2}$ can be used to recognize the presence or absence of the subject (phase F4), as shown in the lower half of Fig. 5

\section{TABLE I. FEATURE LEARNING EXPERIMENT PHASE}

\begin{tabular}{ccl}
\hline Label & $\begin{array}{c}\text { Duration } \\
\text { (second) }\end{array}$ & \multicolumn{1}{c}{ Description } \\
\hline F1 & 5 & Subject sitting before the desk with a good posture \\
F2 & 10 & Subject sitting before the desk with a poor posture \\
F3 & 5 & Subject sitting before the desk with a good posture \\
F4 & 10 & Subject leaves the desk \\
F5 & 10 & Subject returns and sits with a good posture \\
\hline
\end{tabular}
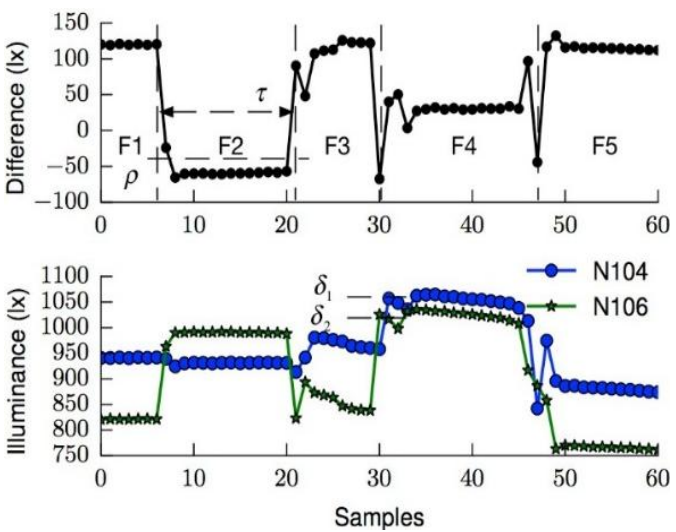

Figure 5. Light sensor signal with five experiment phase

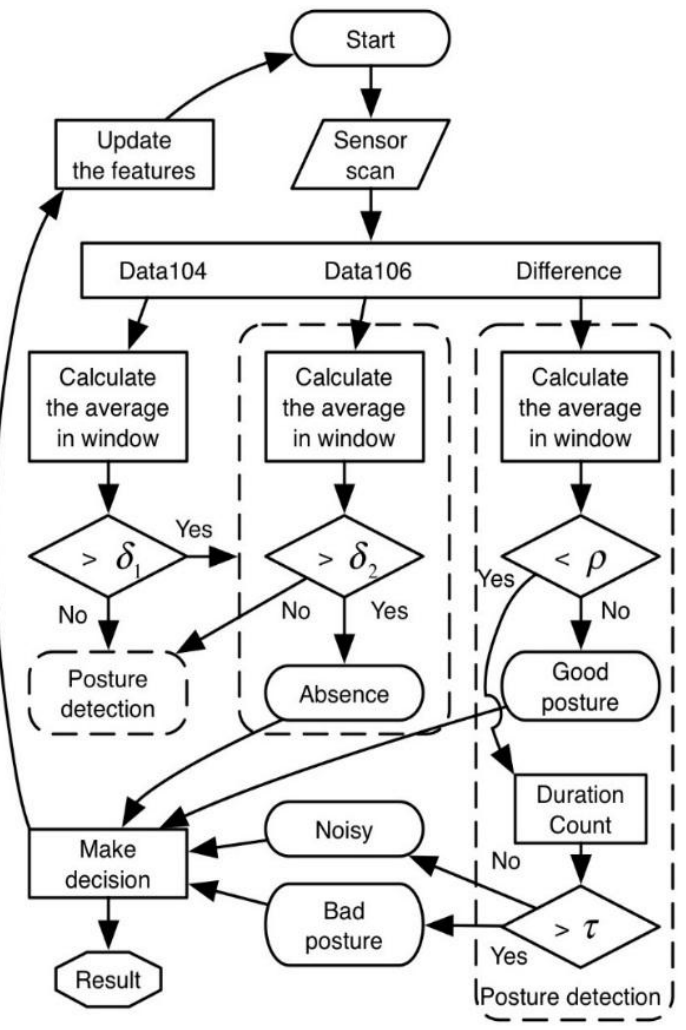

Figure 6. The flowchart of the proposed classifer
There are many different machine learning classification algorithms that could be used, such as Decision Tree (DT), Support Vector Machine (SVM) and Artificial Neural Networks (ANN). Generally, ANN and SVM require a long training time and are subject to problems of over-fitting, however they are able to learn complex relationships between features. By comparison, a DT classifier works well and is quick to train and to classify when relatively simple relationships exist between features.

In our study, a DT based classifier is chosen, and the structure of the feature decisions is constructed by manual inspection of the training data. The flowchart of the classifier is showed in Figure 6. Following the experimental phases as shown in Table I, a training dataset of 100 samples of 60 seconds was obtained. As can be seen by the decision tree in Fig. 6, threshold values of the four features $\delta_{1}, \delta_{2}, \rho$ and $\tau$ are used to make a classification. Initial values of these threshold values are first chosen by visual inspection of the data in Figure 5. Optimized values are the found by a grid search of nearby values of the threshold and evaluating the classification accuracy, as shown in Table II.

TABLE II. FEATURE LEARNED FOR CLASSIFICATION

\begin{tabular}{cccc}
\hline Feature & Initial value & Optimized value & Description \\
\hline$\delta_{1}$ & 1049 & 1030 & Peak threshold 1 \\
$\delta_{2}$ & 1021 & 1010 & Peak threshold 2 \\
$\rho$ & -42 & -40 & Valley threshold \\
$\tau$ & 15 & 15 & Valley duration
\end{tabular}

\section{Evaluation}

After building a classifier based on the training data, we conducted an evaluation experiment based on the proposed light-based system (L) to learn about the system's robustness to several different environment conditions such as cloudy days and noisy incoming light levels. We comparde the performance of our system against the camera based method (C) during the evaluation experiment. In Table III we show the five different environmental conditions used during the evaluation experiment. In each environment we collect data 50 times following the phases shown in Table 1 for 60 seconds each. All the data is stored in the SD card on RPi board and the classification algorithm is run off-line. So there are 3000 samples in each environmental condition. Data is classified for both light and camera methods. Fig. 7 presents the error count and error rate metrics for these three different classes: good posture (GP), poor posture (PP) and absence (A) with 1800, 600 and 600 samples of each class respectively, in each of 5 environments, E1 to E5.

\section{TABLE III. EVALUATION EXPERIMENT ENVIRONMENTS}

\begin{tabular}{ccl}
\hline Environment & Label & \multicolumn{1}{c}{ Description } \\
\hline Daytime & E1 & Sunshine \\
Daytime cloudy & E2 & Cloudy \\
Daytime with noise 1 & E3 & Other subjects pass by \\
Daytime with noise 2 & E4 & Subject reading book/ using phone \\
Daytime with noise 3 & E5 & Subject turns around \\
\hline
\end{tabular}



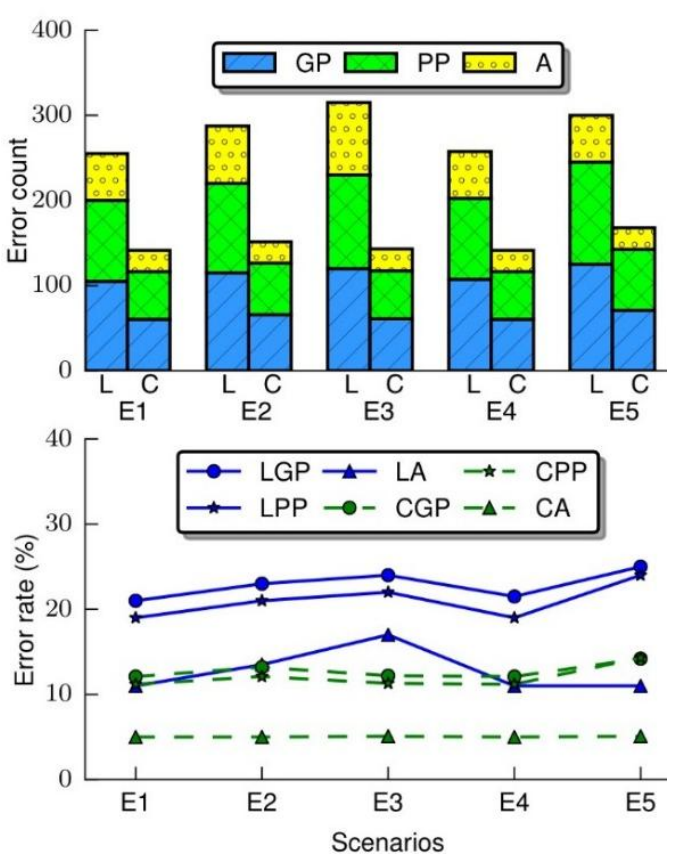

Figure 7. Evaluation results

Compared to the camera based method, the proposed ambient light method shows a higher error rate of about $18 \%$. From the error count figure, it is seen that the error mainly comes from the posture classification (GP and PP). Both systems can classify the absence class with high accuracy. In a cloudy weather condition (E2), the error rate of the proposed system increases by $2 \%$. This is a small increase given that the system was only trained on a sunny day environment. Also other subjects passing by (E3) the system will decrease the accuracy of the system, as this movement causes a light power change around the system. Other activities (E4) has little effect on the proposed system which means that the system is robust against such activities. Finally, the proposed shows poorest accuracy in the E5 environment since this is quite different to the training data.

\section{CONCLUSION}

In this paper, a sitting condition monitoring system based on an ambient light sensor network was developed. We conducted experiments to learn an optimal classifier and evaluate the robustness of the system in five different environments, and the following conclusions were reached.

First, by combining the light power features from different light sensors, a subject's sitting condition can be accurately classified using a simple decision tree classifier. Training data was used to optimize the DT thresholds. Second, our evaluation experiments confirm that the proposed system has an accuracy of around $82 \%$. It can detect an absence class better than the posture classes. It is robust to some noise, with small decreses in accuracy is such cases.

We have identified several important tasks for further investigation. First, we would like to do experiments to learn about an optimal setup of the system, i.e. to vary the number and position of the light sensors. Second, we would like to train the system based on a broader range of environments, not just sunny day data. Third, we would like to set up the system for longer term analysis of more subjects to collect more data about their daily sitting conditions (sitting posture, sitting duration and rest duration), and to study their response to an automatic warning system for poor posture.

\section{REFERENCES}

[1] B. Juul-Kristensen, K. Søgaard, J. Støyer, J. and C. Jensen, "Computer users' risk factors for developing shoulder, elbow and back symptoms." Scandinavian Journal of Work, Environment \& Health, 2004, pp.390-398.

[2] Byeong-Gu Ahn, , Yun-Hong Noh, and Do-Un Jeong, "Smart chair based on multi heart rate detection system," IEEE Sensors Conference, 2015.

[3] Paul Duffy and Alan F. Smeaton, "Measuring the effectiveness of user interventions in improving the seated posture of computer users." Ambient Intelligence, 2013.

[4] Bilge Mutlu,, Andreas Krause, and Jodi Forlizzi, et al, "Robust, lowcost, non-intrusive sensing and recognition of seated postures," User Interface Software and Technology, 2007.

[5] Haruna Ishimatsu, and Ryoko Ueoka, "BITAIKA: development if self posture adjustment system," Proceedings of the $5^{\text {th }}$ Augmented Human International Conference. 2014.

[6] Melody Oliver, Grant M. Schofield, and Hannah M. Badland, et al, "Utility of accelerometer thresholds for classifying sitting in office workers," Preventive Medicine 51.5 ,2010 , pp. 357-360.

[7] Katsumma Tanaka, Shoya Ishimaru, and Koichi Kise, et al, "Nekoze!: monitoring and detecting head posture while working with laptop and mobile phone," International Conference on Pervasive Computing , 2015. 